\title{
Simulation Analysis of 32 Channel WDM System using SMF 28 Optical Fiber at Various Power Levels
}

\author{
Manpreet Kaur \\ M. Tech. Student, \\ Department of Electronic and \\ Communication Engineering, \\ A.C.E.T Amritsar, India
}

\author{
Atul Mahajan \\ Head of Dept., \\ Department of Electronic and \\ Communication Engineering, \\ A.C.E.T Amritsar, India
}

\begin{abstract}
Optical communication in case of WDM system is processing at an astonishing due to high transmission capacity. In this paper, we developed 32 channels WDM system at 40 Gbps is used to improve the system quality at different power levels and frequency spacing at the length of $50 \mathrm{~km}$ using single mode fiber (SMF). EDFA is used for better gain. In this work we enhance the system efficiency and system capacity by using DCF (dispersion compensating fiber). Also, BER an analysis has been shown through simulation at various power levels and frequency levels, the results are shown in terms of BER, Q-factor and Eye height using optisystem 7.0
\end{abstract}

\section{Keywords}

Wavelength division multiplexing (WDM), single mode fiber (SMF), Erbium doped fiber amplifier (EDFA), BER, quality factor (Q-Factor), optisystem 7.0

\section{INTRODUCTION}

Optical communication in case of WDM system is processing at an astonishing rate due to high transmission capacity [1]. In this modern era there is increasing demand for high speed data and Internet services which require more capacity and networking system [2]. So, to increase the capacity of system we have new technology i.e WDM system, WDM system uses multiple wavelengths to transmit over a single fiber [3]. Before the transmission it is very important to investigate the parameters of the system. In long distance of optical signals faces two challenges attenuation and dispersion. To overcome this problem we use Erbium doped amplifiers (EDFA) and dispersion compensating fiber (DCF). EDFA (erbium doped fiber amplifier) is extensively used because of its compatibility with optical fiber and also for its low insertion loss and high gain [4]. The EDFA operates on wave band of $1550 \mathrm{~nm}$. Fiber also suffers from dispersion due to fiber material ,so dispersion has to be minimized by some methods but installing the $\mathrm{DCF}$ (dispersion compensating fiber) is one of the methods to compensate the dispersion due to SMF (single mode fiber) and also DCF are more mature and not easily effected by wide bandwidth and temperature [5,6]. For increase the transmission capacity of optical communication system there some basis methods first is by increasing spectral efficiency, second is by increasing the bandwidth of optical fiber the [7]. The third method is by increasing various power levels and frequency spacing is used in this work.

In this paper, we have simulate the 32 channel 40 Gbps WDM system having central frequency $190 \mathrm{THz}$ at various power levels and frequency spacing. The performance of parameters are evaluating by using optisystem 7.0.

The paper has been organized as follow: section 2 begins with
System model modification, section 3 describes the simulation setup section, results and discussion analysis at various power levels and frequency spacing in section 4 , section 5 concludes the findings.

\section{SYSTEM MODEL MODIFICATION}

In this work, modified 32 channel WDM system. Dispersion compensating fiber is use to mitigate the effect of losses and dispersion in SMF for various power levels and at various frequency spacing. The system model of WDM system is shown in fig 1 .

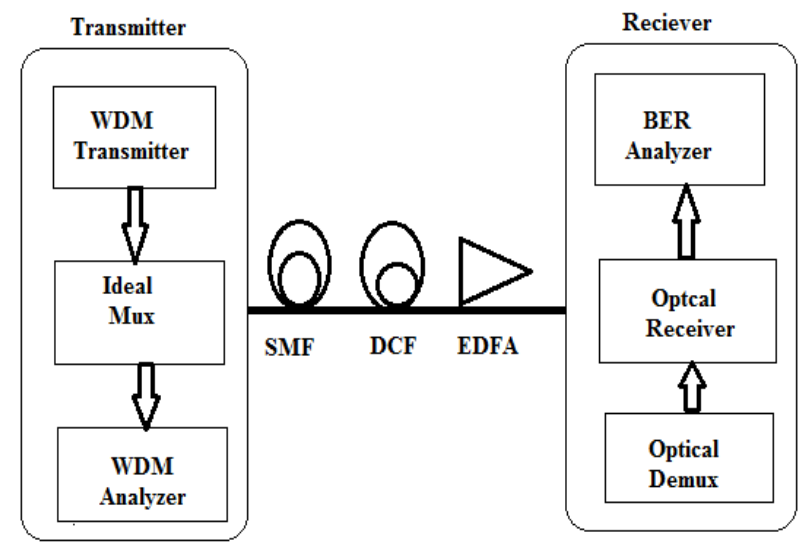

Fig.1: Block diagram of WDM system

Any communication system abides of three basic sections: Transmitter, communication channel and receiver section $[8$, 9]. In this system the transmitter section consists of three components WDM transmitter, ideal MUX and WDM analyzer. The WDM system transmitter produces laser signals at specific wavelength. The multiplexer couples together different wavelength then combine from transmission into SMF. The EDFA is use for better gain, the EDFA are used for long distance. DCF (dispersion compensating fiber) is used to reduce the effect of dispersion having attenuation $0.5 \mathrm{db} / \mathrm{km}$. The receive side employs demultiplexer to distribute the optical signal to wavelength selectors. Optical receiver is used to receive the optical signal and BER analyzer is used to visualize the simulation results in terms of q-factor, BER etc.

\section{SIMULATION SETUP MODEL}

In this model, 32 channels are transmitted at 40 Gbps speed at various power levels and frequency spacing. The WDM system is designed in optisystem V7.0. In this model, there are main three sections: transmitter section, communication channel and receiver section. The WDM transmitter having wavelength range of 1530 to $1550 \mathrm{~nm}$, operating at Frequency 
of $190 \mathrm{THz}$. The communication section consists of single mode fiber (SMF) having length of $50 \mathrm{~km}$ and with attenuation of $0.2 \mathrm{db} / \mathrm{km}$. Erbium doped fiber amplifier (EDFA) is capable of amplifying multiple signals on different wavelength with gain $10 \mathrm{db}$. DCF (dispersion compensating fiber) is used to mitigate the effect of dispersion. The simulation results are obtained in terms of Q- factor, BER, Eye height etc using optisystem 7.0. The simulation model is shown in figure 2 .

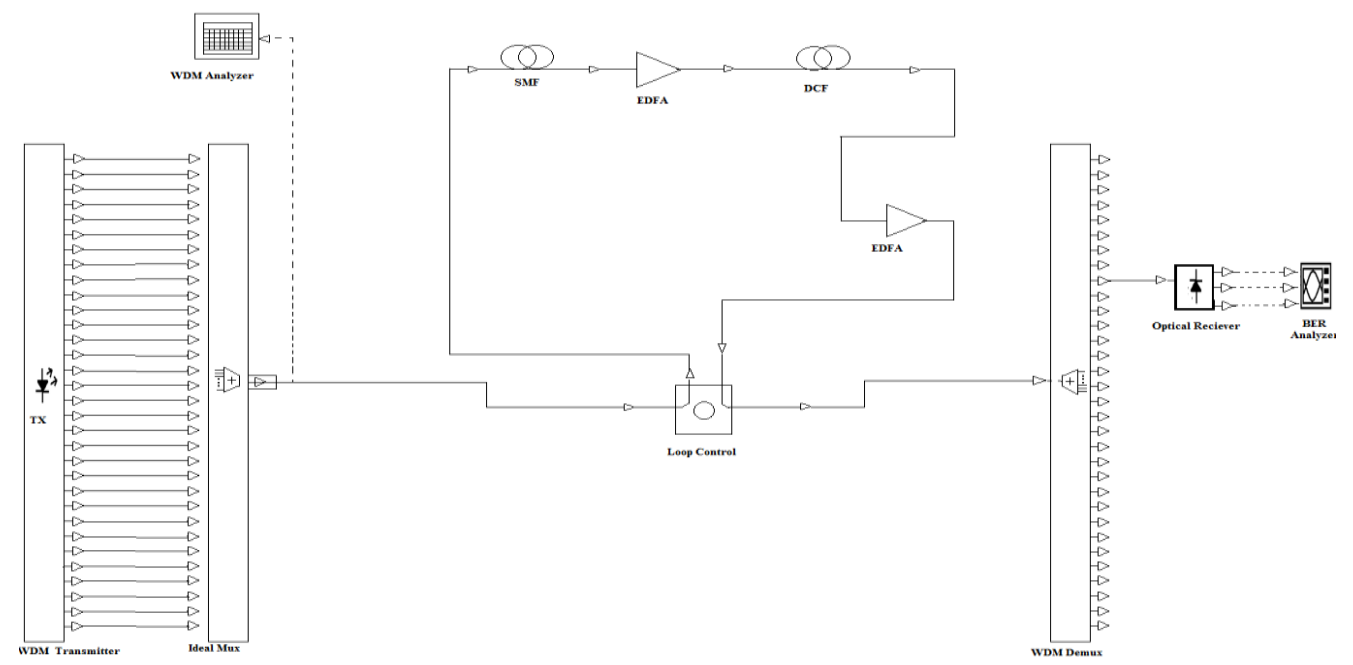

Fig.2: Simulation setup model 32 channel WDM system

\section{RESULT AND DISCUSSION}

In this section, analyze the effect of optical signal when it passes through the SMF. To analyze the effect of various Power levels and frequency spacing use the BER analyzer. The results are shown in terms of Q- factor, BER, Eye height etc.

\subsection{BER analysis at various power levels}

Now, move towards the analysis of WDM system at various power levels of single mode fiber: To increases system quality and vary the power level from $-7 \mathrm{dBm}$ to $7 \mathrm{dBm}$ perform analysis.

\subsubsection{Analysis at-7dBm input power}

When the input power of optical source is set at $-7 \mathrm{dBm}$, then the eye diagram with maximum quality factor, BER, eye height etc. is shown in figure 3.

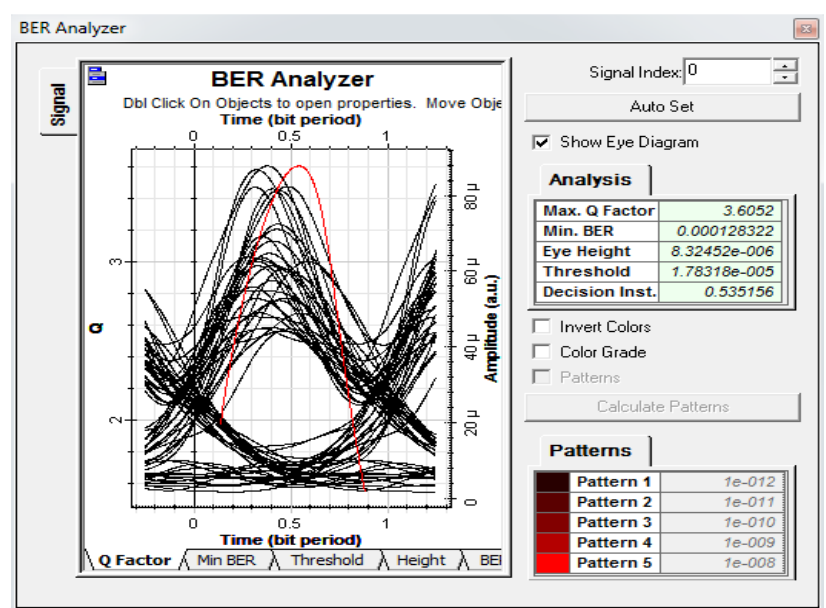

Fig.3: Eye diagram (BER analyzer at $\mathbf{- 7} \mathbf{~ d B m}$ )

In figure 3 the maximum quality factor is 3.6052 and BER is 0.000128322 when the input launch power is $-7 \mathrm{dBm}$.

\subsubsection{Analysis at -2dBm input power}

Now increase the input power and set at $-2 \mathrm{dBm}$, then the eye diagram with maximum Q-factor and minimum BER, shown in figure 4.

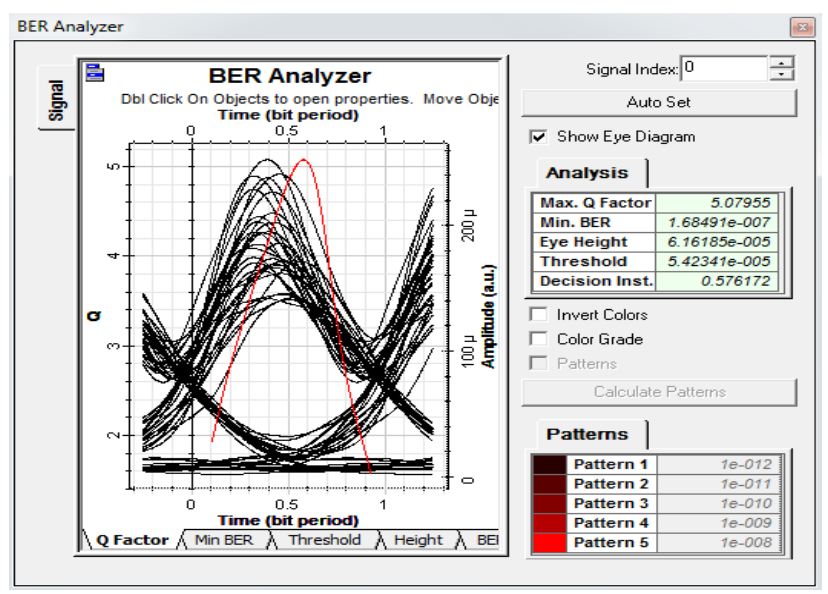

Fig.4: Eye diagram (BER analyzer at -2 dBm)

From figure it is shown that the q factor 5.07955 is increased by increasing the power level and minimum BER is 1.68491e007.

\subsubsection{Analysis at 3dBm input power}

Again, increases the input power up to $3 \mathrm{dBm}$ the maximum Q-factor and minimum BER is shown in figure 5. 


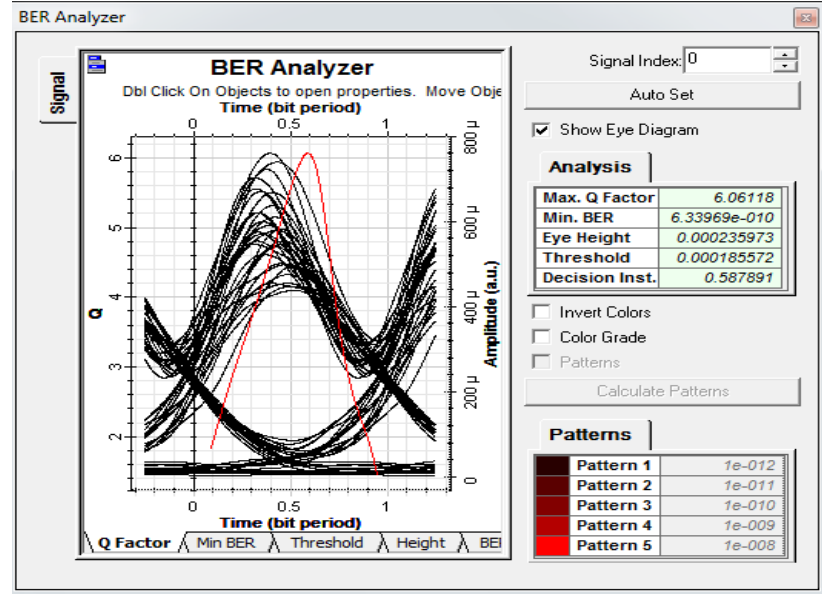

Fig.5: Eye diagram (BER analyzer at $3 \mathrm{dBm}$ )

In this case the quality factor 6.06118 is increased and BER is $6.33969 \mathrm{e}-10$.

\subsubsection{Analysis at $7 \mathrm{dBm}$ input power}

Further increased the input power and set at $7 \mathrm{dBm}$ the maximum quality factor and BER is shown in figure 6.

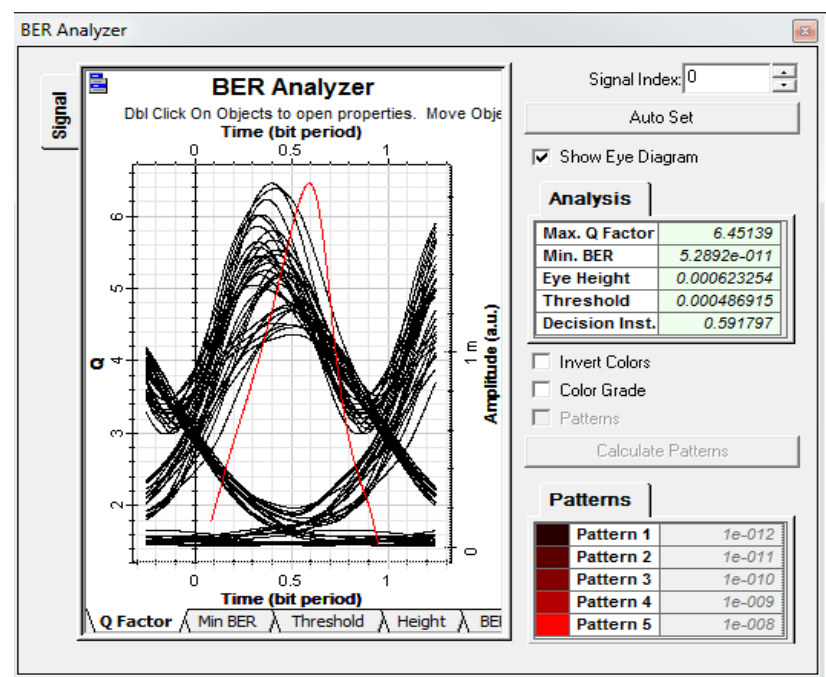

Fig.6: Eye diagram (BER analyzer at $7 \mathrm{dBm}$ )

From figure 6 it is clearly shown that the Quality factor is increased to 6.45139 .

By comparing figure 3, 4, 5 and 6 analyze that the maximum quality factor is increases with the increase in input power. So, the overall capacity of system is increased.

\subsection{BER analysis at different frequency spacing}

In this section, analyze the WDM system at different frequency spacing: Figure 7, figure 8 and figure 9 shows the BER analysis at different frequency spacing or the overall system quality.

Firstly analyze the system at frequency spacing of $200 \mathrm{GHz}$, the maximum quality factor and BER is as Shown in figure 7.

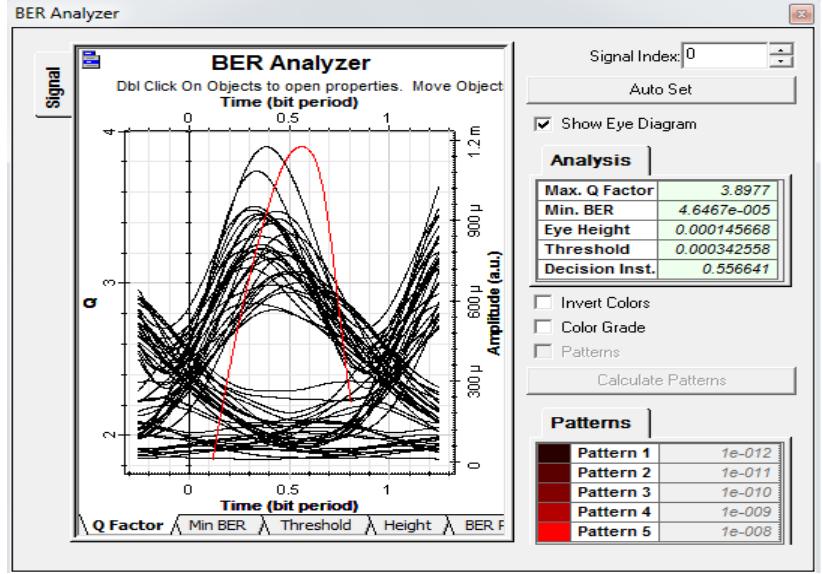

Fig.7: Eye diagram (BER analyzer at $190 \mathrm{GHz}$ )

At 190 the quality factor is 3.8977. The decreases the frequency spacing of system, now the minimum BER and Qfactor is shown in figure 8 .

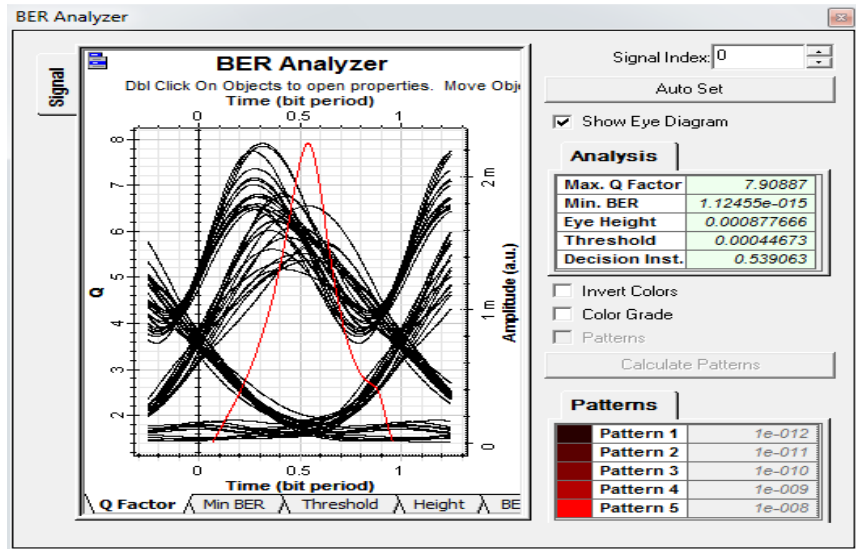

Fig.8: Eye diagram (BER analyzer at $170 \mathrm{GHz}$ )

It is shown from figure 8 that with the decrease in frequency spacing the Q- factor increases it means the overall quality of system is increases.

Further, again decreases the frequency spacing and set at 150 $\mathrm{GHz}$, minimum BER and maximum Q-factor is as shown in figure 9 .

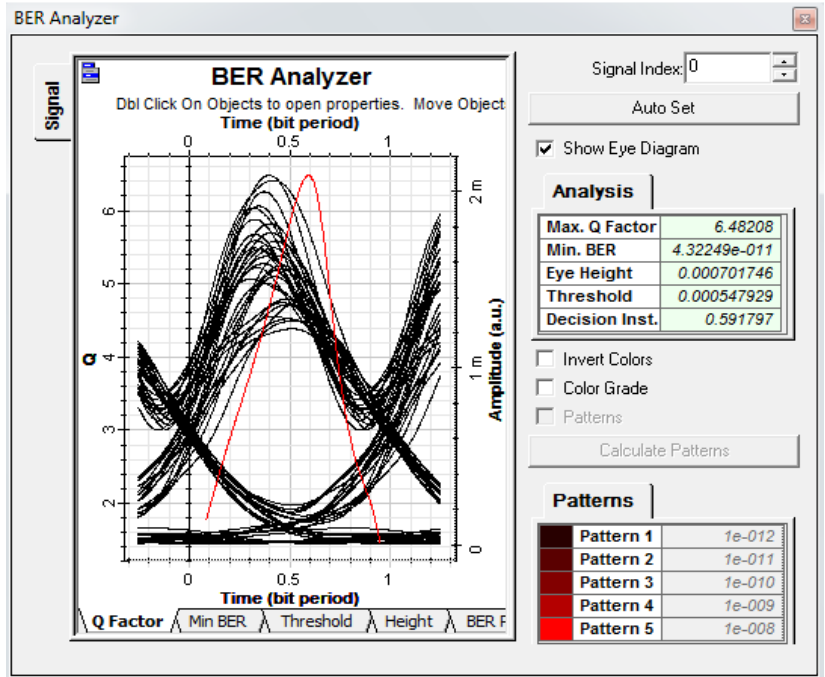

Fig.9: Eye diagram (BER analyzer at $150 \mathrm{GHz}$ ) 
From figure 9 it is shown that Q- factor again decreases at frequency spacing $150 \mathrm{GHz}$.

From simulation, achieve better results at frequency spacing of $170 \mathrm{GHz}$ at quality factor 7.90887 .

\section{CONCLUSION}

In this work, single channel is replace into 32 channels using SMF, and achieve better performance in case of system quality at various power levels and frequency spacing. The better performance of WDM system has been observed with quality factor of 6.48208 in case of power levels and 7.90887 in case of frequency spacing. The results are shown through Eye diagram; it shows better values of Quality factor and minimum BER.

\section{AKNOWLEDGEMENT}

I want to thank to my guide Atul Mahajan head of department (E.C.E) at Amritsar College of Engineering and Technology, Amritsar for providing help and guidance.

\section{REFERENCES}

[1] Charvi Mittal, Yuvraj Singh Rathore, Sonakshi Verma "Reducing SRS and FWM in DWDM systems" International Journal of Advanced Scientific and Technical Research, 2014.

[2] Tianjiao Xie, Muhammad Asif, Husan Ali, H.M.Rehan Afzal "Reparation of Chromatic Dispersion Using Dispersion Compensation Bank and bit-Error Rate Analysis at Various Power Levels in 40 Gbps Fiber Optics System" $7^{\text {th }}$ IEEE International Congress on Image and Signal Processing, 2014.

[3] Parveen Bagga, Himali Sarangal"Simulation of $32 \times 20$ Gb/s WDM and DWDM System at Different Dispersion" International Journal of Advanced Research in Computer and Communication Engineering, 2015.
[4] V. S. Lavanya*, V. K. Vaidyan "Optimized Flattened Gain Spectrum in C -Band WDM using Automatic Gain Control in Bi-Directionally Pumped EDFA" International Journal of Engineering Research \& Technology,2015.

[5] Prateeksha Sharma, Mr. Bipan Koushal, Shrija Jain"Performance Analysis Of Dispersion Compensation Of Optical Fiber Using EDFA" International Journal of Engineering Research \& Technology, 2013.

[6] Gurpreet Kau1,Navdeep Kaur"Use of Dispersion Compensating Fiber in Optical Transmission Network for NRZ Modulation Format" International Journal Of Engineering And Computer Science,2014.

[7] Neha Chaudhary, Ashish Kumar, Priya Upadhaye, Gagandeep Kaur" Evaluating the 32*45Gbps Transmission over SMF link Based on DWDM/EDFA Architecture" International Journal of Emerging Technology and Advanced Engineering, 2015.

[8] Mishal Singla, Preeti, Sanjiv Kumar" Comparative Analysis of EDFA based 32 channels WDM system for bidirectional and counter pumping techniques" Comparative Analysis of EDFA based 32 channels WDM system for bidirectional and counter pumping techniques, 2014 .

[9] Mehtab Singh, and Rajveer"Investigation of EDFA based 32 Channel C-Band Optical WDM System for Different Pumping Techniques" International Journal of Signal Processing, Image Processing and Pattern Recognition, 2015. 